\title{
Effect of Chemical Reaction in a Third Grade Fluid in Porous Medium analyzed by Unsteady Magnetohydrodynamic Couette
}

\author{
*IDOWU, AS; USMAN, S \\ Department of Mathematics, University of Ilorin, Kwara State, Nigeria \\ *Corresponding Author Email: $\underline{\text { asidowu@gmail.com, princemagee1@gmail.com }}$
}

\begin{abstract}
An analysis was carried out on unsteady magnetohydrodynamic (MHD) Couette third grade fluid in a porous medium in the presence of chemical reaction parameter. The Darcy's law and Fick's law of diffusion were introduced to stimulate the porosity and chemical reaction in the problem. The resulting flow problem was investigated and model of the problem in form of partial differential equations were obtained. He-Laplace method was used to obtain the solution of the equations and expressions for the velocity field, temperature and concentration distribution were given for each case. Graphical results for various interesting parameters were analyzed and presented.
\end{abstract}

\section{DOI: https://dx.doi.org/10.4314/jasem.v22i6.12}

Copyright: Copyright (c) 2018 Idowu and Usman. This is an open access article distributed under the Creative Commons Attribution License (CCL), which permits unrestricted use, distribution, and reproduction in any medium, provided the original work is properly cited.

Dates: Received: 24 April 2018; Revised: 19 June: 2018; Accepted: 30 June 2018

Keywords: Plane Couette; Magnetohydrodynamic; Porous Medium; Chemical Reaction Parameter.

The study of flow of non-Newtonian fluid in a porous medium has drawn considerable interest in recent years due to its wide applications in technology and in several industrial manufacturing processes such as in the drilling of oil and gas well, polymer sheet extrusion from dye, glass fibber and paper production e.t.c. Constitutive equations that can be used to describe such fluids have been proposed. Some recent attempts may be mentioned in the references.

The steady MHD coquette flow with heat transfer of an electrically conducting fluid was studied considering the ion slip by Attia, (2005). It was found that the increase in the ion slip parameter increases $u$ but decreases $w$ while on the other hand, increasing the hall parameter increases the velocity component $u$ and w. Also the ion slip or the viscosity exponent has a marked effect on the axiel and transverse component of the skin friction and the Nusselt number at both walls of the channel. Attia, (2008), looked at unsteady MHD couette flow with heat transfer in the presence of uniform suction and injection. It is of interest to see that the magnetic field on the temperature at the center of the channel depends on time, where for small time, increasing the magnetic field increases the temperature and for large time, increasing the magnetic field decreases the temperature. An analysis for flow and heat transfer characteristics in a third grade fluid between two porous plates was studied by Hayat et al., (2008), and observed the effects of weak magnetic field and porosity on both velocity and temperature profiles with the boundary layer thickness decreasing with increasing parameters $\mathrm{N}$ and $\varepsilon$. Das et al., (2009), looked at the unsteady hydromagnetic couette flow of a viscous incompressible electrically conducting fluid in a rotating system and it was found that for large time primary velocity decreases with increase in the rotating parameter $\mathrm{k} 2$ while it increases with increase in the magnetic parameter $\mathrm{m} 2$. Attia et al., (2011), studied the unsteady flow with heat transfer of a dust conducting fluid under the influence of an applied uniform magnetic field in the presence of uniform suction and injection and an exponential decaying pressure gradient. It was observed that the suction velocity has a more apparent effect than the magnetic field on the steady state time of the velocity and temperature of the dust particles. Effect of inclined Hartmann in an unsteady MHD couette flow between two infinite parallel porous plates in an inclined magnetic field was studied by Simon, (2014), where increase in the Prandtl number decreases the temperature distribution and increase in the radiation parameter increases the temperature distribution. Sreekala and Reddy, (2014), examined the steady MHD couette flow of an incompressible viscous fluid through a porous medium between two infinite parallel plates under effects of inclined magnetic field and saw that the velocity components enhances with increasing the Reynolds number, suction parameter and Darcy parameter while decreases with increasing the Hartmann number. The steady MHD couette flow between two infinite parallel plates in a uniform 
transverse magnetic field was studied by Kiema et al., where increase in the magnetic field strength and magnetic inclinations results into decrease in the velocity profiles. Considering the above literature the unsteady variable were never considered as well as the medium of flow to be porous together with chemical reactions. Thus this study is important.

Mathematical Formulation: Considering the unsteady MHD Couette flow of a third grade fluid between two infinite parallel plate distance $h$ apart. The upper plate and lower plate are at a distance $h$ apart of a rectangular system with the $x$-axis as the flow direction. The upper plate is assumed to be moving with constant speed $U$ and the lower plate kept stationary. The temperature of the higher and lower plate are $\theta_{1}$ and $\theta_{\circ}$ respectively. We consider MHD Couette unidirectional flow with zero pressure gradient and a uniform applied magnetic field $B$ 。 acting in the $y$-direction. Thus, we have $\theta(y, t), V[u(y, t), 0,0]$ and $C(y, t)$. The continuity equation of motion, heat equation and mass equations in the presence of a chemical reaction were taken into account. The boundary layer equation governing the flow are

$\frac{\partial u}{\partial y}=0$

$\rho \frac{\partial u}{\partial t}=\mu \frac{\partial^{2} u}{\partial y^{2}}+\alpha_{1}\left(\frac{\partial^{3} u}{\partial y^{2} \partial t}\right)+6 \beta_{3}\left(\frac{\partial u}{\partial y}\right)^{2} \frac{\partial^{2} u}{\partial y^{2}}-\sigma B_{\circ}^{2} u+R_{x}$

$\rho C_{p} \frac{\partial \theta}{\partial t}=k_{1} \frac{\partial^{2} \theta}{\partial y^{2}}+\mu\left(\frac{\partial u}{\partial y}\right)^{2}+\alpha_{1}\left(\frac{\partial^{3} u}{\partial y^{2} \partial t}\right)+2 \beta_{3}\left(\frac{\partial u}{\partial y}\right)^{4}$

$\frac{\partial C}{\partial t}=D \frac{\partial^{2} C}{\partial y^{2}}-K_{r} C$

The porosity medium is presented in the momentum equation via the $R_{x}$ term. The effects of the pores on the velocity field is incorporated by implementing Darcy's resistance in its generalized form. For Darcy's law, we have

$$
R_{x}=\nabla P=-\frac{\phi}{k} \text { (apparent viscosity) } \mathrm{V}
$$

Where $\phi$ is the porosity of the porous medium, $k$ the permeability of the porous medium, using equation (5) in (2) gives the equation

$\rho \frac{\partial u}{\partial t}=\mu \frac{\partial^{2} u}{\partial y^{2}}+\alpha_{1}\left(\frac{\partial^{3} u}{\partial y^{2} \partial t}\right)+6 \beta_{3}\left(\frac{\partial u}{\partial y}\right)^{2} \frac{\partial^{2} u}{\partial y^{2}}-\frac{\phi}{k}[\mu u+$

$\left.\alpha_{1}\left(\frac{\partial u}{\partial t}\right)+2 \beta_{3} u\left(\frac{\partial u}{\partial y}\right)^{2}\right]-\sigma B_{\circ}^{2} u$

Also from the fick's second law of diffusion equation (4) was obtained.

Here, $u$ is the velocity in the $y$ direction, $\theta$ is the temperature, $C$ is the concentration, $\rho$ is the fluid density, $\alpha_{1}$ and $\beta_{3}$ are material constants, $D$ is the diffusion coefficient of the diffusing species, $k_{1}$ the thermal conductivity, $C_{p}$ the specific heat, $\mu$ the coefficient of viscosity and $K_{r}$ is the chemical reaction parameter studied between the two plates.

The appropriate conditions are

$$
\begin{aligned}
& u(h, t)=u_{\circ} \exp (-t) \\
& u(\infty, t)=0 \\
& u(y, t)=u_{\circ} \exp (-y) \\
& \theta(h, t)=u_{\circ} \exp (-t) \\
& \theta(y, t)=u_{\circ} \exp (-y) \\
& C(h, t)=u_{\circ} \exp (-t) \\
& C(y, t)=u_{\circ} \exp (-y)
\end{aligned}
$$

Upon making use of the following non-dimensional variables

$$
\begin{aligned}
& \bar{u}=\frac{u}{u_{\circ}}, \bar{y}=\frac{u_{\circ} y}{v}, \bar{t}=\frac{u_{\circ}^{2} t}{v}, \overline{\alpha_{1}}=\frac{\alpha_{1} u_{\circ}^{2}}{\rho v^{2}}, \overline{\beta_{3}}=\frac{3 \beta_{3} u_{\circ}{ }^{4}}{\rho v^{3}}, \\
& \bar{\phi}=\frac{\phi v^{2}}{k u_{\circ}{ }^{2}}, \overline{M^{2}}=\frac{\sigma B^{2} v}{\rho u_{\circ}^{2}}, \bar{\theta}=\frac{\theta-\theta_{\circ}}{\theta_{1}-\theta_{\circ}}, \bar{C}=\frac{C-C_{\circ}}{C_{1}-C_{\circ}}
\end{aligned}
$$

The above definition of velocity satisfies the continuity equation and equations (3), (4), (6), (7), (8) and (9) can be written as

$$
\begin{gathered}
\rho \frac{\partial u}{\partial t}=\epsilon \frac{\partial^{2} u}{\partial y^{2}}+\alpha_{*}\left(\frac{\partial^{3} u}{\partial y^{2} \partial t}\right)+6 \beta\left(\frac{\partial u}{\partial y}\right)^{2} \frac{\partial^{2} u}{\partial y^{2}}-\phi_{*} u- \\
\beta_{*} u\left(\frac{\partial u}{\partial y}\right)^{2}-M_{*}^{2} u \\
\frac{\partial \theta}{\partial t}=\frac{1}{P r} \frac{\partial^{2} \theta}{\partial y^{2}}+E c\left(\frac{\partial u}{\partial y}\right)^{2}+\alpha\left(\frac{\partial^{3} u}{\partial y^{2} \partial t}\right)+\beta_{\circ}\left(\frac{\partial u}{\partial y}\right)^{4}
\end{gathered}
$$

$\frac{\partial C}{\partial t}=D_{*} \frac{\partial^{2} C}{\partial y^{2}}-K r_{*} C-M$

$u(0, t)=\exp (-t)$

$u(\infty, t)=0$

$u(y, 0)=\exp (-y)$

$\theta(0, t)=\exp (-t)$

$\theta(y, 0)=\exp (-y)$

$C(0, t)=\exp (-t)$

$C(y, 0)=\exp (-y)$

Where

$\epsilon=\frac{1}{1+\alpha_{1} \phi}, \alpha_{*}=\frac{\alpha_{1}}{1+\alpha_{1} \phi}, \beta=\frac{3 \beta_{3}}{1+\alpha_{1} \phi}, \phi_{*}=\frac{\beta_{3} \phi}{1+\alpha_{1} \phi}, M_{*}{ }^{2}=$ $\frac{M^{2}}{1+\alpha_{1} \phi}, \frac{1}{P r}=\frac{\alpha}{v}, E C=\frac{u_{\circ}{ }^{2}}{C_{p}\left(\theta_{1}-\theta_{\circ}\right)}$, 
$\alpha=\frac{\alpha_{1} u_{\circ}}{C_{p}\left(\theta_{1}-\theta_{\circ}\right)}=\frac{\alpha_{1}}{u_{\circ}} \times \frac{u_{\circ}{ }^{2}}{C_{p}\left(\theta_{1}-\theta_{\circ}\right)}=\frac{\alpha_{1 E c}}{u_{\circ}} \quad, \quad \beta_{\circ}=\frac{\beta_{3} u_{\circ}{ }^{2}}{C_{p}\left(\theta_{1}-\theta_{\circ}\right)}=$ $\frac{\beta_{3} k_{1}}{\mu C_{p}} \times \frac{\mu u_{\circ}^{2}}{k_{1}\left(\theta_{1}-\theta_{\circ}\right)}=\frac{\beta_{3} B r}{P r}, D_{*}=D v$,

$K r_{*}=\frac{K r u_{0}^{2}}{v}, M=\frac{K r u_{0}^{2}}{v} \times \frac{C_{\circ}}{\left(C_{1}-C_{0}\right)}$.

With $\operatorname{Pr}$ and $E c$ the respective Prandtl and Eckert numbers, $\phi_{*}$ porosity of the porous medium, $D_{*}$ diffusion coefficient, $K r_{*}$ chemical reaction parameter, $M_{*}$ magnetic field parameter.

For He-Laplace method, the solutions for the velocity distributions $u(y, t)$, temperature field $\theta(y, t)$ and the concentration field $C(y, t)$ are expressed as follows

$$
\begin{aligned}
u_{\circ}(y, t) & =\exp (-y) \\
& u_{1}(y, t)=\left(\epsilon-\phi_{*}-M_{*}{ }^{2}\right) \exp (-y) t+(\beta-
\end{aligned}
$$$$
\left.\beta_{*}\right) \exp (-3 y) t
$$

$\theta \circ(y, t)$

$=\exp (-y)+E c \exp (-2 y) t$

$+\left(\epsilon-\phi_{*}-M_{*}^{2}\right) E c \exp (-y) t^{2} 3(\beta$

$$
(-4 y) t^{2}+2\left(\beta-\beta_{*}\right)
$$

$\left.-\beta_{*}\right) E c \exp \left(\epsilon-\phi_{*}-M_{*}^{2}\right) \operatorname{Ec} \exp (-4 y) t^{3}+$

$$
\frac{\left(\epsilon-\phi_{*}-M_{*}^{2}\right)^{2} E c \exp (-4 y) t^{3}}{3}
$$

$+3\left(\beta-\beta_{*}\right)^{2} E c \exp (-6 y) t^{3}$

$+\left(\epsilon-\phi_{*}-M_{*}^{2}\right) \alpha \exp (-y) t+9\left(\beta-\beta_{*}\right) \alpha \exp (-3 \mathrm{y}) \mathrm{t}$

$+\beta_{\circ} \exp (-4 y) t+2\left(\epsilon-\phi_{*}-M_{*}{ }^{2}\right) \beta_{0} \exp (-4 y) t^{2}$

$+6\left(\beta-\beta_{*}\right) \beta_{\circ} \exp (-6 y) t^{2}$

$+12\left(\beta-\beta_{*}\right)\left(\epsilon-\phi_{*}-M_{*}{ }^{2}\right) \beta_{\circ} \exp (-6 y) t^{3}$

$+2\left(\epsilon-\phi_{*}-M_{*}^{2}\right)^{2} \beta_{\circ} \exp (-4 y) t^{3}$

$+18\left(\beta-\beta_{*}\right)^{2} \beta_{\circ} \exp (-8 y) t^{3}$

$+9\left(\beta-\beta_{*}\right)\left(\epsilon-\phi_{*}-M_{*}^{2}\right)^{2} \beta_{0} \exp (-6 y) t^{4}$

$+27\left(\beta-\beta_{*}\right)^{2}\left(\epsilon-\phi_{*}-M_{*}^{2}\right) \beta_{\circ} \exp (-8 y) t^{4}$

$+\left(\epsilon-\phi_{*}-M_{*}^{2}\right)^{3} \beta_{0} \exp (-4 y) t^{4}$

$+27\left(\beta-\beta_{*}\right)^{3} \beta_{\circ} \exp (-10 y) t^{4}$

$+\frac{\left(\beta-\beta_{*}\right)\left(\epsilon-\phi_{*}-M_{*}{ }^{2}\right)^{3} \beta_{0} \exp (-6 y) t^{5}}{5}$

$+\frac{54\left(\beta-\beta_{*}\right)^{2}\left(\epsilon-\phi_{*}-M_{*}^{2}\right)^{2} \beta_{\circ} \exp (-8 y) t^{5}}{3}$

$+\frac{108\left(\beta-\beta_{*}\right)^{3}\left(\epsilon-\phi_{*}-M_{*}^{2}\right) \beta_{0} \exp (-10 y) t^{5}}{5}$

$+\left(\epsilon-\phi_{*}-M_{*}^{2}\right)^{4} \beta_{0} \exp (-4 y) t^{5}$

$+\frac{12\left(\beta-\beta_{*}\right)^{4} \beta_{\circ} \exp (-12 y) t^{5}}{5}$

$$
\begin{aligned}
& \theta_{1}(y, t) \\
& =\frac{\exp (-y) t}{\operatorname{Pr}}+\frac{2 E c \exp (-2 y) t^{2}}{\operatorname{Pr}} \\
& +\frac{4\left(\epsilon-\phi_{*}-M_{*}^{2}\right) E c \exp (-2 y) t^{3}}{3 P r}+\frac{16\left(\beta-\beta_{*}\right) E c \exp (-4 \mathrm{y}) t^{3}}{\operatorname{Pr}} \\
& +\frac{8\left(\beta-\beta_{*}\right)\left(\epsilon-\phi_{*}-M_{*}{ }^{2}\right) \operatorname{Ec} \exp (-4 y) t^{4}}{\operatorname{Pr}} \\
& +\frac{\left(\epsilon-\phi_{*}-M_{*}{ }^{2}\right)^{2} E c \exp (-2 y) t^{4}}{3 P r}+\frac{27\left(\beta-\beta_{*}\right)^{2} E c \exp (-6 y) t^{4}}{P r} \\
& +\frac{\left(\epsilon-\phi_{*}-M_{*}^{2}\right) \alpha \exp (-y) t^{2}}{2 P r}+\frac{81\left(\beta-\beta_{*}\right) \alpha \exp (-3 y) t^{2}}{2 P r} \\
& +\frac{8 \beta_{0} \exp (-4 y) t^{2}}{\operatorname{Pr}}+\frac{32\left(\epsilon-\phi_{*}-M_{*}{ }^{2}\right) \beta_{\circ} \exp (-4 y) t^{3}}{3 \operatorname{Pr}} \\
& +\frac{72\left(\beta-\beta_{*}\right) \beta_{0} \exp (-6 y) t^{3}}{\operatorname{Pr}} \\
& +\frac{108\left(\beta-\beta_{*}\right)\left(\epsilon-\phi_{*}-M_{*}^{2}\right) \beta_{\circ} \exp (-6 y) t^{4}}{P r} \\
& +\frac{8\left(\epsilon-\phi_{*}-M_{*}{ }^{2}\right)^{2} \beta_{\circ} \exp (-4 y) t^{4}}{\operatorname{Pr}} \\
& +\frac{288\left(\beta-\beta_{*}\right)^{2} \beta_{\circ} \exp (-8 y) t^{4}}{\operatorname{Pr}} \\
& +\frac{324\left(\beta-\beta_{*}\right)\left(\epsilon-\phi_{*}-M_{*}{ }^{2}\right)^{2} \beta_{\circ} \exp (-6 y) t^{5}}{5 P r} \\
& +\frac{1728\left(\beta-\beta_{*}\right)^{2}\left(\epsilon-\phi_{*}-M_{*}{ }^{2}\right) \beta_{\circ} \exp (-8 y) t^{5}}{5 P r} \\
& +\frac{16\left(\epsilon-\phi_{*}-M_{*}{ }^{2}\right)^{3} \beta_{\circ} \exp (-4 y) t^{5}}{5 P r} \\
& +\frac{540\left(\beta-\beta_{*}\right)^{3} \beta_{\circ} \exp (-10 y) t^{5}}{\operatorname{Pr}} \\
& +\frac{72\left(\beta-\beta_{*}\right)\left(\epsilon-\phi_{*}-M_{*}{ }^{2}\right)^{3} \beta_{\circ} \exp (-6 y) t^{6}}{5 P r} \\
& +\frac{576\left(\beta-\beta_{*}\right)^{2}\left(\epsilon-\phi_{*}-M_{*}{ }^{2}\right)^{2} \beta_{0} \exp (-8 y) t^{6}}{5 \operatorname{Pr}} \\
& +\frac{360\left(\beta-\beta_{*}\right)^{3}\left(\epsilon-\phi_{*}-M_{*}{ }^{2}\right) \beta_{\circ} \exp (-10 y) t^{6}}{P r} \\
& +\frac{8\left(\epsilon-\phi_{*}-M_{*}^{2}\right)^{4} \beta_{\circ} \exp (-4 y) t^{6}}{15 P r} \\
& +\frac{1944\left(\beta-\beta_{*}\right)^{4} \beta_{\circ} \exp (-12 y) t^{6}}{5 \operatorname{Pr}}
\end{aligned}
$$

\section{RESULTS AND DISCUSSION}

This section is made to show physical features of the variables like $\left(\phi_{*}\right)$ porosity term, $\left(M_{*}\right)$ magnetic term, (Ec) Eckert number, (Pr) Prandtl number, $\left(D_{*}\right)$ diffusion coefficient and $\left(K r_{*}\right)$ chemical reaction parameter on velocity, temperature and concentration distributions. From figures 1-6, which indicates the velocity, temperature and concentration profiles showing the influence of various parameters on them. Figures 1-2 indicates the effects of porosity parameter $\phi_{*}$, and the magnetic field parameter, $M_{*}$ on the velocity $u$. It was observed that the effects of $\phi_{*}$ and $M_{*}$ are similar, showing that velocity decreases by increasing $\phi_{*}$ and $M_{*}$ respectively. 


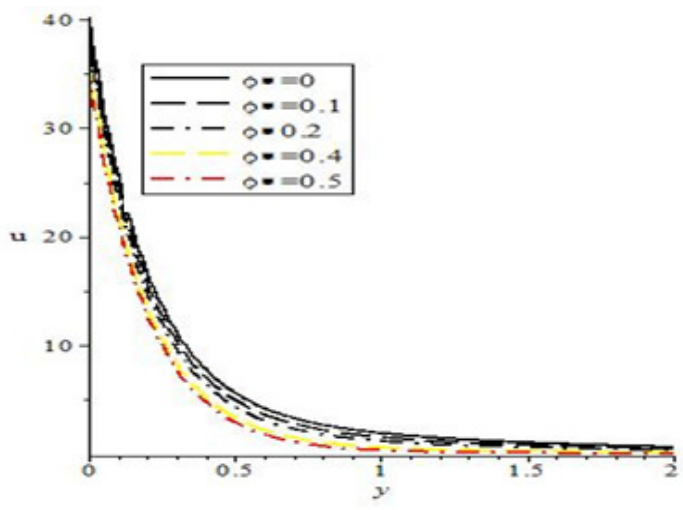

Fig 1: Influence of $\phi_{*}$ on velocity $(u)$ when $M_{*}=0.5, t=$ $1.5, \beta_{*}=0.1, \beta=0.1, \epsilon=1, \alpha_{*}=1$

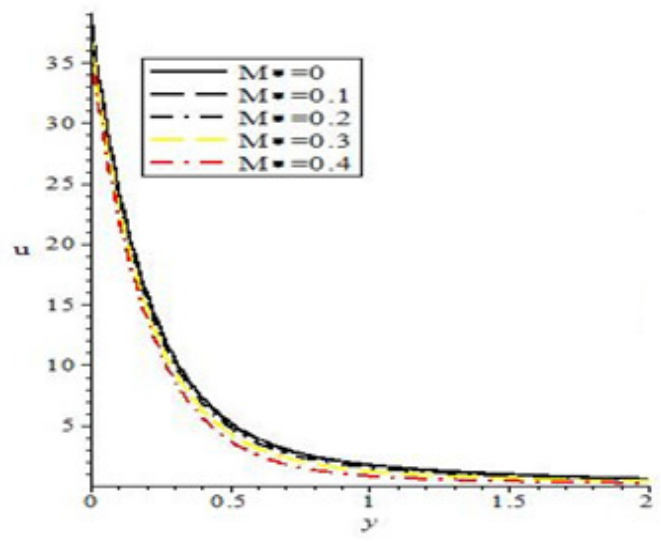

Fig 2: Influence of $M_{*}$ on velocity $(u)$ when $t=1.5, \phi_{*}=0.5, \beta_{*}=$ $0.1, \beta=0.1, \epsilon=1, \alpha_{*}=1$

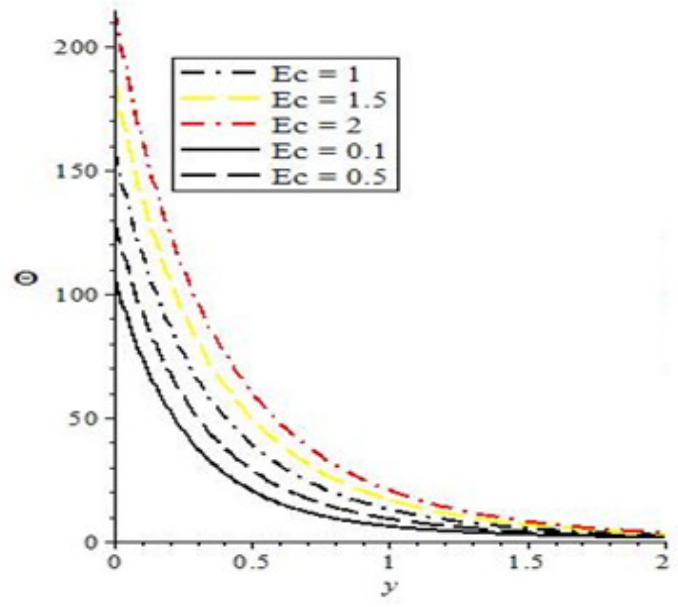

Fig 3: Influence of $E c$ on Temperature $(\theta)$ when $M_{*}=0.5, \phi_{*}=$ $0.5, \beta_{\circ}=0.1, \beta_{*}=0.1, \beta=0.1, \epsilon=1, \alpha=1, \operatorname{Pr}=0.5, t=1.5$

Thus implies that porosity and magnetic parameter reduces the velocity of the fuild in the flow. The higher the values of the parameter the smaller the value of velocity. Thus, porosity and magnetic terms brings reduction in the velocity of the fluid. In figures 3-4, the effect of $E c$ and $\operatorname{Pr}$ on temperature $\theta$ was presented. It is seen that the temperature increases and the thermal boundary thickness decreases with increase in $E c$ number while the temperature decreases and the thermal boundary thickness increases with increasing $\operatorname{Pr}$ number.



Fig 4: Influence of $\operatorname{Pr}$ on Temperature $(\theta)$ when $M_{*}=0.5, \phi_{*}=$ $0.5, \beta_{\circ}=0.1, \beta_{*}=0.1, \beta=0.1, \epsilon=1, \alpha=1 E c=0.5, t=1.5$

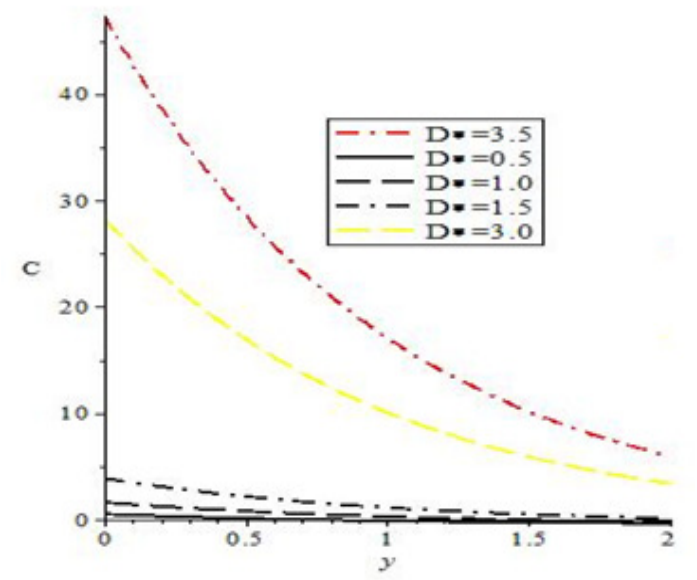

Fig 5: Influence of $D_{*}$ on Concentration $(C)$ when $M=0.5, t=$ $1.5, K r_{*}=1.5$

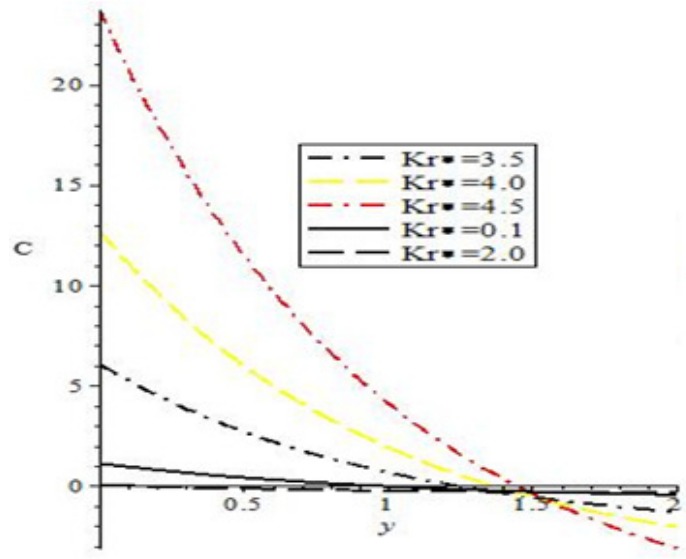

Fig 6: Influence of $K r_{*}$ on Concentration $(C)$ when $M=0.5, t=$ $1.5, D_{*}=0.5$ 
Figures $5-6$ are sketched to describe the variations of the diffusion coefficient of the diffusing species $D_{*}$ and the chemical reaction parameter $K r_{*}$ on the concentration field. However, $\mathrm{C}$ decreases upon increasing $D_{*}$ and while increase in chemical reaction parameter increases the concentration of the fluid.

Conclusion: Unsteady MHD Couette flow of a third grade fluid in a porous medium in the presence of chemical reaction parameter is analyzed using HeLaplace method. We modified Hayat et al., (2008), and observed the effects of porosity parameter term, Prandtl number and chemical reaction parameter on the velocity, temperature and concentration profiles and found that the boundary layer thickness increases with increase in porosity parameter term and Prandtl number while increases the concentration of the field with increase in the chemical reaction parameter.

\section{REFERENCES}

Attia, HA (2005). MHD Couette Flow with Temperature Dependent Viscosity and the Ion Slip. T. J. Sci. Eng. 8(1): 11-16.

Attia, HA (2008). Unsteady MHD Couette Flow with Heat Transfer in the Presence of Uniform Suction and Injection. Mech. Mech. Eng. 12(2): 165-176.

Attia, HA; Al-kaisy, AMA; Ewis, KM (2011). MHD Couette Flow and Heat Transfer of a Dusty Fluid with exponential Decaying Pressure Gradient. $T$. J. Sci. Eng. 14(2): 91-96.
Das, S; Maji, SL; Guria, M; Jana, RN (2009). Unsteady MHD Couette Flow in a Rotating System. Math. Comp. Mod. 50: 1211-1217.

Hayat, T; Ellahi, R; Mahomed, FM (2008). The Analytical Solution for Magnetohydrodynamic Flow of a Third Grade Fluid in a Porous Medium. Z. Natureforsch. 64a: 531-539.

Kiema, DW; Mangonge, WA; Bitok, JK; Adenyah, RK; Barasa, JS (2015). On the Steady MHD Couette Flow Between Two Infinite Parallel Plates In a Uniform Transverse Magnetic Field. $J$. Appl. Math. Bioinf. 5(1): 87-99.

Simon, D (2014). Effect of Heat of Transfer on Unsteady MHD Couette Flow between Two Infinite Parallel Porous Plates in an Inclined Magnetic Field. Inter. J. Math. Stat. In. 2(6): 2321-4767.

Sreekala, L; Reddy, EK (2014). Steady MHD Couette of an Incompressible viscous Fluid through a Porous Medium between Two Infinite Parallel Plates under effect of Incline Magnetic Field. Inter. J. Eng. Sci. 3(9): 18-37. 